\title{
Young People's Conceptualization of Their Wellbeing: Culturally Situated Understandings in the Context of Kazakhstan
}

\author{
Eva Brown Hajdukova, Liz Winter, Ros McLellan \\ Faculty of Education, University of Cambridge, Cambridge, UK \\ Email: eva.brownhajdukova@gmail.com
}

How to cite this paper: Hajdukova, E.B., Winter, L. and McLellan, R. (2017) Young People's Conceptualization of Their Wellbeing: Culturally Situated Understandings in the Context of Kazakhstan. Health, 9, 1542-1566.

https://doi.org/10.4236/health.2017.911114

Received: September 1, 2017

Accepted: October 27, 2017

Published: October 30, 2017

Copyright $\odot 2017$ by authors and Scientific Research Publishing Inc. This work is licensed under the Creative Commons Attribution International License (CC BY 4.0). http://creativecommons.org/licenses/by/4.0/

\begin{abstract}
Little is known about the wellbeing of young people living in post-Soviet nation contexts such as Kazakhstan. The qualitative data reported represents the views of 309 Kazakhstani young people on their conceptualizations of wellbeing that fell broadly into three major thematic categories: external factors from one's surroundings; relationships with others; and internal aspects of the self. The direct consequence of inadequate physical environment on health and the environment's impact on psychological wellbeing through leisure, lifestyle, education, and employment opportunities were emphasized. Affirmative connections with others were associated with increased levels of emotional wellbeing. The importance of making independent choices as well as being actively involved in leisure, volunteering and extracurricular school activities for achieving optimal levels of wellbeing was highlighted.
\end{abstract}

\section{Keywords}

Wellbeing, Health, Kazakhstan, Young people

\section{Introduction}

Interest in young people's wellbeing has been steadily increasing across political, social and educational contexts as reflected by attempts at conceptualization, definition and quantification through the burgeoning number of internationally comparable indicators and frameworks [1] [2] [3]. An increased attention towards the monitoring of young people's wellbeing can also be found at the policy level, especially after the United Nations developed the Convention on the Rights of the Child in 1989. Since this declaration, there have been numerous international calls to not only include young people's wellbeing in systematic 
and detailed statistical national accounts [4] [5] [6] [7], but also to develop positively framed youth wellbeing indicators suitable across developmental trajectories [8].

It has been widely recognized that a strong sense of wellbeing enables young people to engage positively and confidently with their environments and therefore to take full advantage of learning opportunities [9]. While the importance of young people's wellbeing on effective learning and positive development is undisputed, there are still conflicting perspectives on what young people's wellbeing actually looks like and how to effectively measure it [10] [11]. However, it should be noted that the concept of wellbeing has often been discussed in the literature as an "elusive" concept challenging to define [12]. These difficulties have been commonly linked to the nature of wellbeing as a multidimensional phenomenon, making it difficult to encapsulate in a brief theory and hence to be operationalized as a universal measurable quantity [10]. Another critical contributing factor to the apparent challenge in defining wellbeing may be its multidisciplinary nature, with the concept of wellbeing being commonly described as an umbrella term that encompasses an array of specific indicators and concepts [13] with no agreed definition across disciplines [14].

Moreover, when seeking to understand the complexities of wellbeing, the importance of cultural context is inherent in any description of wellbeing as a socially contingent construct embedded in culture and prone to change and redefinition over time, both in terms of changes in individual's life course as well as socio-cultural context [10] [14] [15]. Therefore, when considering the relevance of wellbeing for different individuals and groups, wellbeing measures and policies need to be continually reassessed and redefined in order to ensure that they are still meeting the wellbeing needs of the society in question [16].

The research project presented in this article attempted to capture young people's conceptualization of their wellbeing by utilizing qualitative, young people-focused research methodology. This kind of research not only affirms young people as competent social actors, and therefore valid source of information [15], but also emphasizes cultural context, thus enabling for better capture of local understandings and the diversity of meanings young people give to wellbeing [17]. More importantly, little is known about the wellbeing of children and young people living in post-Soviet nations contexts such as Kazakhstan [18] and even less is known about how political and educational reform has impacted on generations brought up under the very different regimes of capitalism and communism [19]. Therefore the aim of this paper is to add to the existing body of knowledge on young people's wellbeing by utilizing the case of Kazakhstan as an illustrative example of a post-Soviet context of non-Western traditions and culture.

\section{Kazakhstani Context}

Kazakhstan declared its independence from the Soviet Union in 1991 and joined 
the Commonwealth of Independent States. Since then it has undergone a significant economic transformation by actively engaging in a wide range of political reforms, which include a modernization agenda to improve public service provision, with increased focus on educational reform [20]. These wide-reaching and ongoing social changes in life conditions and lifestyles have also transformed values. This period of economic prosperity has contributed to the transition from a collectivist approach to individualism, which is complicated and controversial in Kazakhstani traditional society, especially for the older generation [21]. Research suggests that younger cohorts are more interested in personal competitiveness, careers, entrepreneurship and freedom of conviction, all values characteristic in Western societies, which coexist with certain collectivist traditional historical and cultural features in the minds of younger generation [22]. Mukh$\operatorname{tar}$ [22] further argues that despite these transformations, in the culture of contemporary Kazakhstan family, labor, and prosperity still come forefront which allow for the blurring of boundaries between traditional and individualistic values. In addition, a socioeconomic crisis has engulfed Kazakhstan in the past three years and has considerably affected young people's health and wellbeing, especially those who are most vulnerable [23].

\section{Young People's Views on Wellbeing}

Increasingly, young people's views have been recognized as providing a unique perspective of the world and positively contributing towards the better monitoring of key issues affecting their lives, while enabling the more effective development of student centered quality supports, services and policies [24]. However, this has not always been the case as research into young people's wellbeing has been widely appropriated as a way of capturing adult aspirations for young people [25]. Adults' views have traditionally informed policy and practice, with parents often considered to be the primary voice of their child [15]. It has been argued that this adult-centered approach has led to the development of definitions and theories of young people's wellbeing that are often conceptions that convey adults' understanding of young people's wellbeing as opposed to young people's own constructions of their wellbeing [26]. There has also been little acknowledgement that children's and young people's perspectives are different and separate from those of adults, thus young people have been rendered as "objects" rather than active participants [27]. However, emerging evidence has highlighted that children and young people do not necessarily share adults' views on what is important to them and their wellbeing, further highlighting that we cannot solely rely on adults' inferences [14] [26] [28]. The inclusion of young people's perspectives in all stages of research has also been identified as a crucial step in building a solid foundation on which to develop data collection instruments that are relevant and meaningful to young people, potentially enabling the development of more valid and reliable measures and indicators of young people's wellbeing [25]. So far, no research has attempted to explore what wellbeing means 
for young people in Kazakhstan; therefore rather than take an assumptive air of what young people need, the project reported in this paper sought to explore young people's perspectives on their wellbeing directly to determine how young people in Kazakhstan conceptualize and define the complex issue of wellbeing.

\section{The Study}

This paper draws on data collected as part of a wider mixed-methods collaborative research project that lasted two years (April 2015 - June 2017) and was organized in four phases. Qualitative data that were collected in phases one and two from 49 mixed-gender focus groups ( 5 - 7 participants) from grades 9 (between the ages of 13 - 14 years), 10 (between the ages 15 - 16 years) and 11 (between the ages of $17-18$ years) will form the focus of this paper. All participants were selected on a voluntary basis either by the research team members or class teachers to ensure a willingness to participate. As the research was conducted in mainstream schools during lessons, the grades and thus participants were selected based on their availability. It is also important to note that the qualitative data reported on in this paper were obtained from four distinct school clusters representing the range of schools that exist in Kazakhstan in order to provide a more comprehensive picture of young people's wellbeing. Cluster I (CI) represents selective secondary schools. The non-selective secondary schools were then divided into the three following clusters: cluster II (CII) (urban mainstream secondary schools), cluster III (CIII) (rural mainstream secondary schools), and cluster IV (CIV) (multi-graded small village schools where students from different age groups are educated in the same class and are a particular feature of the Kazakhstani educational system in sparsely populated areas). The data were drawn from eleven different locations and included 29 secondary schools, covering south, east, west, north-west, north, and central Kazakhstan (for more detail please see the Table 1 below).

Table 1. Methodological and ethical considerations.

\begin{tabular}{|c|c|c|c|}
\hline & $\begin{array}{l}\text { School location and number } \\
\text { of schools in each location }\end{array}$ & $\begin{array}{l}\text { Focus Groups } \\
\text { (2-7 people) }\end{array}$ & $\begin{array}{l}\text { Number of group } \\
\text { interviews in each cluster }\end{array}$ \\
\hline \multirow{5}{*}{$\begin{array}{l}\text { Phase 1-May 2015: scoping visit to conduct first } \\
\text { inquiry into how wellbeing is conceived and managed }\end{array}$} & Almaty (3) & Mixed Grade (1) & Cluster I (5) \\
\hline & Shymkent (2) & Grade 9 (1) & Cluster II (2) \\
\hline & Astana (2) & Grade 10 (1) & Cluster III (1) \\
\hline & Pavlodar (2) & Grade 11 (6) & Cluster IV (1) \\
\hline & $\Sigma=9$ & $\Sigma=9$ & $\Sigma=9$ \\
\hline \multirow{8}{*}{$\begin{array}{l}\text { Phase 2-November 2015: more detailed inquiry } \\
\text { into the constructs of wellbeing in Kazakhstan }\end{array}$} & Akmola (3) & Grade 9 (8) & Cluster I (12) \\
\hline & Atyrau (6) & Grade $9 \& 10(7)$ & Cluster II (21) \\
\hline & Kokshetau (1) & Grade 10 (7) & Cluster III (6) \\
\hline & Kostanay (1) & Grade 11 (16) & Cluster IV (1) \\
\hline & Oskemen (4) & Grade $12(2)$ & \\
\hline & Shymkent (6) & & \\
\hline & Zhambyl (1) & & \\
\hline & $\Sigma=22$ & $\Sigma=40$ & $\Sigma=40$ \\
\hline
\end{tabular}


The researchers abided by the ethical guidelines of the British Educational Research Association (BERA) and the British Psychological Society (BPS) in order to apply ethical and research integrity. Ethical approval was further granted according to procedural requirements and via the relevant routes for both universities involved in the collaborative research project-in the UK and Kazakhstan. All participants were informed about the research, their rights, and what their potential participation would entail. They were also assured of anonymity and confidentiality alongside the right to withdraw at any stage before each interview took place. Permission was sought to record (digitally) the conversations and, once assent was granted, the recording began with a reminder not to mention people by name if possible. Assurances were also given regarding confidentiality and access to the data and its safe storage. All transcribed data were anonymized prior to analysis and translators employed to generate transcripts in English were required to destroy all digital copies of the data other than the end transcripts and to sign confidentiality agreements. Participants were free to converse in Kazakh, Russian or English as they preferred with live translation provided to and from English. The request for consent was also given in the chosen language.

Focus groups were chosen as the primary method of data collection as they have the potential to reveal narrative representations of social experience and elucidate the meanings they have for the speaker [29]. The use of focus groups also enabled the creation of data from multiple voices, while allowing the researchers to capture young people's views by uncovering those aspects of understanding that often remain hidden in the more conventional in-depth interviewing method [30]. Despite the reported strengths of focus groups, it is important to consider some of the limitations of this method. These include the tendency for certain types of socially acceptable opinions to emerge and for certain types of participants to dominate the research process, therefore it is crucial to analyze focus group data in ways which do not ignore these limitations [29].

The research team consisted of one group facilitator (one of the seven primary researchers) and one translator. The translators were local Kazakh and Russian speaking $\mathrm{PhD}$ students with excellent knowledge of both English and the subject area. During the group interviews, the participants responded to a series of open-ended questions based on the interview protocol developed in a collaborative process between the researchers. The interview guide included dominant themes such as relationships, hobbies, health and environment, identified in preliminary research encounters and from extant literature on the topic of wellbeing [30]. During the interviews, in-depth dialogue was encouraged concerning the significance of the various themes from each participant's perspective. The implementation of such an approach also enabled the process of data collection to be systematic and comprehensive while simultaneously allowing the freedom to be situated as fairly naturalistic and conversational. It is also important to note that the translators were also involved in the validation of the interview 
protocol for its cultural appropriateness.

Moreover, it has been argued that the use of translators in the research process is of epistemological consequence as the translators themselves form a part of the process of knowledge production. Also, the power relationship between the researcher and the translator needs to be considered as the researcher often relies on the translator's perspective to a certain extent [31]. In this study, however, translators played an important part in the process of knowledge production. They were actively engaged in ongoing debate and reflection on the interview process and often provided feedback based on which appropriate adaptations to the respondent's cultural context could be made. More importantly, their exchange of local knowledge provided a basis for the development of more culturally sensitive and aware research into young people's wellbeing. Furthermore, as the intention was to learn about young people's perspectives and experiences, researchers made sure that they used words and phrases that were familiar and developmentally appropriate for each age group in order to safeguard opportunities for free expression in ways that were most meaningful or familiar to the participants [32].

The most critical methodological issues stem from the questions of how to introduce and the concept of "wellbeing" to young people in Kazakhstan and how to select or design methods that will elicit their perspectives on this abstract concept. We began the focus groups by asking our participants to pictorially represent or, if preferred, to write down, five things that make people of their age feel good and good about themselves plus five things that make people feel the opposite. The rationale was to make salient and invoke thinking on the two primary aspects of wellbeing of hedonism (feeling good) and eudemonism (functioning well) in simplified terms. The drawings were therefore used in an exploratory manner as an elicitation tool to discover how young people in Kazakhstan conceptualize wellbeing and what they consider to be important factors influencing their wellbeing, with an intent to elude imposing adult-centered concerns [33]. By implementing the drawing activity, participants were encouraged to be more actively involved in the research, while also allowing them time to think about what they wished to portray and what message they would like to convey. The use of drawings in research can provide other benefits as well. The image can be changed and added to, hence participants are more likely to experience better control over the form of expression, unlike an interview situation where responses tend to be quicker and more immediate [32]. Discussions between individual participants and researchers about the meanings of their creations after completion helped to continue to give prominence to the young people's interpretations of wellbeing. This stage also allowed rapport to be built between the researchers and participants, hence mitigating the unequal power relations between young people and adults [33].

Substituting the term "wellbeing" was vital, as many young people were not familiar with this term due to challenges in its literal translation into Russian 
and Kazakh. In both languages the notion of wellbeing tends to imply two meanings; "good health" and "financial prosperity". Hence the decision was made to substitute the term "wellbeing" with a term that referenced how the person felt such as "happy". However, asking young people what makes them "happy" and "unhappy" may focus attention on "fun" and "affect" at the expense of a focus on a deeper internal feeling, state or experience [32]. In order to mitigate the risk of simply recording a hedonistic transitory mood state and also to trigger functionality (eudemonism), researchers also used probing questions asking participants for concepts or elements that were associated with a "good day" and a "bad day".

\section{Data Analysis}

Interview transcripts were analyzed and coded in Nvivo 10 which provided a valuable tool for analyzing the interviews thematically [32] [34]. Thematic analysis was chosen as a primary method for identifying, analyzing, and reporting patterns within the data and for its compatibility with the constructionist paradigm [35]. The themes within the data came from open coding of data without a pre-existing framework [32]. Responses were analyzed using the thematic analysis procedure as described by Braun and Clarke [35]. First, the interview transcripts were carefully read and re-read in order to identify meaningful units of text relevant to the research topic. Secondly, units of text dealing with the same topic were grouped together in analytic categories. It is important to note that, if appropriate, the same unit of text could be included in more than one category. Thirdly, the data were systematically revisited to ensure that a named theme (node) had all data relevant to support the topic included.

In addition, this study's thematic analytical approach was derived from Braun and Clarke's view of coding as a flexible, active, and reflexive process that inevitably bears the mark of the researcher(s), further suggesting that there is no "accurate" way to code data, hence the logic behind inter-rater reliability vanishes [35]. However, in order to ensure that the nuances unique to Kazakhstani culture were captured and represented appropriately in the data interpretation process, the decision was made to conduct further data analysis by randomly selecting $10 \%$ of the transcripts already coded by a non-Kazakh researcher to be coded by a native Kazakh on the team. The coding was then compared and only a few slight variances were identified. These variances were minimal, often the result of differing word choice, and did not prevent the capture of the exact or very similar essence of the selected text.

\section{Kazakhstani Young People's Conceptualization(s) of Wellbeing}

In this study, young peoples' conceptualization(s) of wellbeing fell broadly into three major thematic categories: external factors from one's surroundings; relationships with others, and internal aspects of the self. This is consistent with the 
literature that suggests that these three elements of environment, inter- and intra-personal factors are essential when considering the wellbeing of young people [3] [24] [36] [37], further highlighting the ambiguous and multifaceted nature of the construct of wellbeing [15]. The thematic units identified in this study were consistently reflected in the data, providing a basis for a conceptual framework for wellbeing as identified by the young people in Kazakhstan. This took on a trio of representative factors that moved from external to interactional and then internal facets (summarized in Figure 1 below). It should be also noted, that even though different aspects of school context and school related factors such as school engagement, teachers, peer support, parent involvement and perceptions of academic autonomy were identified and associated with various aspects of the participants' wellbeing, it is not within the scope of this article to report on these findings. These results will be reported in a separate article.

In the following section, the results and discussion are presented together, along with supporting exemplar quotations from focus groups. In order to maintain anonymity whilst still providing a comprehensive picture of findings in terms of personal background; participants' gender, school-grade, school cluster and geographic location are provided for each quote.

\section{External Factors from One's Surroundings}

This first element to wellbeing was generally beyond the personal control of individuals and was a consequence where they lived and of what resources they had to hand to deal with their circumstances. Environments that young people most commonly talked about were their homes, schools, and local areas. These sites where described as places where they lived day-to-day lives, and where their

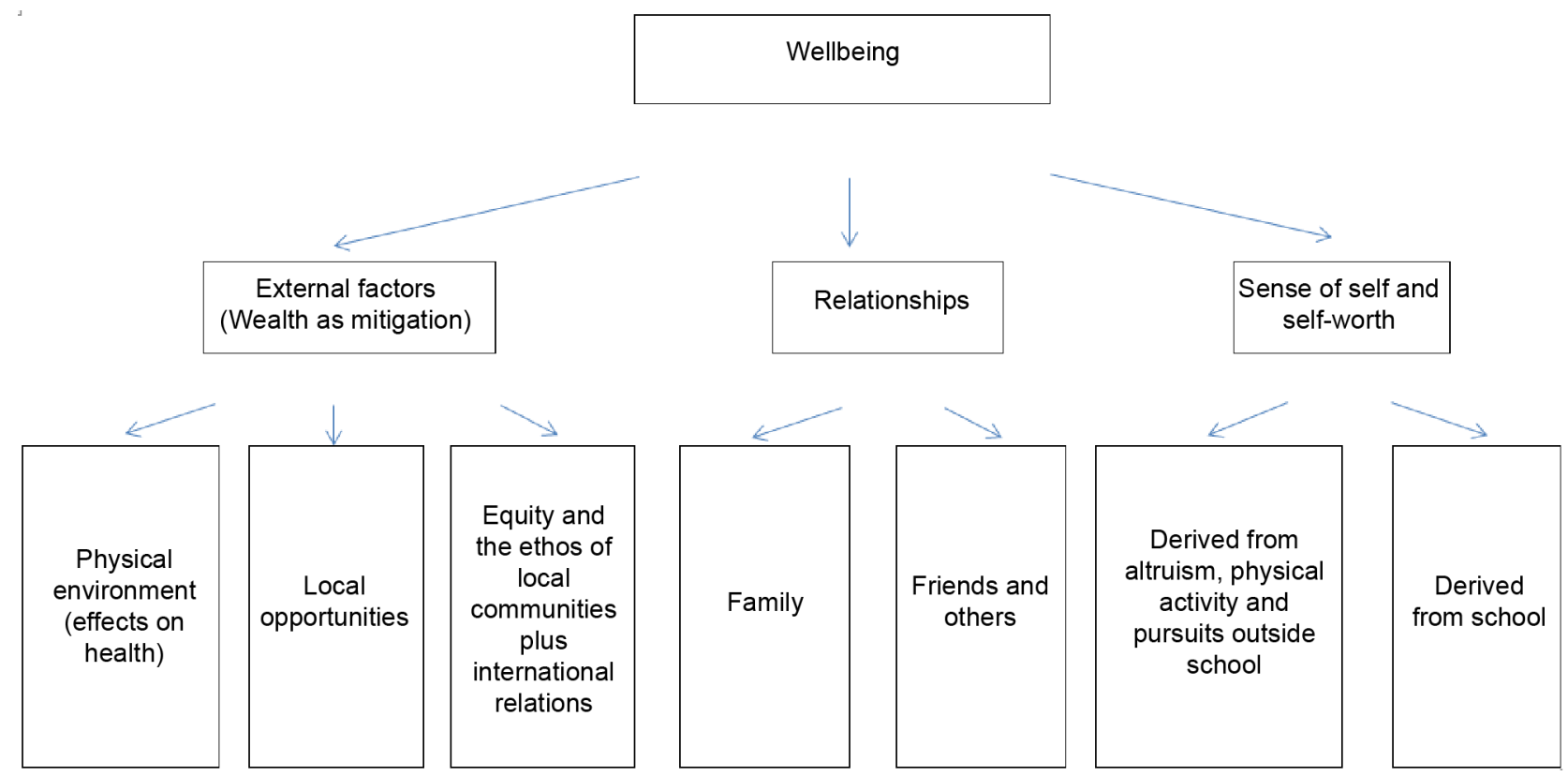

Figure 1. Summary of major themes emergent from young people's own constructs on their conceptualization of wellbeing in Kazakhstan. 
physical and emotional domains coincided. The physical environment, as described by young people in this study, included: land; air; water; plants; animals; buildings; other infrastructure; and resources that may provide opportunities for social and economic development. The social environment was described in terms of layers which started with concrete local issues of personal safety resulting from the local environment and school (tempered by the material resources available to mitigate any effects) and which then moved on to discuss more abstract concerns of international conflict and terrorism stemming from media reporting and educational initiatives. The discourse on external factors can therefore be split into four strands: firstly, the direct consequence on physical wellbeing from the environment as it affects health; secondly, the environment's impact on psychological wellbeing through current leisure, lifestyle and education opportunities alongside future employment prospects; thirdly, real and imminent concerns over personal safety and, fourthly, more abstract concerns over personal and national security. A final part to this section on external factors to wellbeing considers how material wealth interacts with the environment and opportunities alongside perceived matters of social equity such as the effects of widespread corruption.

\subsection{Physical Environment and Health}

Many young people expressed concerns about their own health, as well as the health of their family members and friends. Personal good health status was identified as an important factor influencing optimal functioning and emotional wellbeing. This is clearly a recurrent feature in the literature and one of the mainstay factors in most large-scale research initiatives regarding wellbeing [1] [6] [38] [39] [40].

If you are healthy, you can manage to do anything. If I get ill, yes, I lose my self-regulation skills and time management skills of course. (Male, G9, CI, Oskemen city, east Kazakhstan)

I think the first thing (factor promoting wellbeing) is health. I mean both mental and physical health. I think that when we say "happy" we mean thankful for something like I don't have serious illness. (Female, G10, CII, Shymkent city, south Kazakhstan)

The majority of participants perceived a clean, safe, and healthy environment as vital for achieving optimal physical and emotional wellbeing. However, many young people who lived in industrial, mining, and oil and gas producing cities in Kazakhstan reported serious environmental issues. Air and water pollution, hostile landscape, extreme weather conditions, as well as flooding caused by flash floods and lack of drainage in urban areas were commonly linked by participants in this study to physical harm, emotional distress, and limited engagement in social activities outside school. Exposure to pollution in Kazakhstan is still posing serious health and environmental consequences, particularly in urban and highly industrialized areas, consequently leading to increased mortality, aggra- 
vation of respiratory and cardiovascular diseases, hospital admissions, school absences, and restricted activity days for many young people in Kazakhstan [41]. These concerns are also echoed in the recent OECD multidimensional review of Kazakhstan that states that, even though overall quality of life in Kazakhstan has improved significantly over the last decade, health outcomes still remain a great challenge, and may remain so, unless a strong stimulus is given by the government to improving environmental conditions [42]. To take this further and to generate insight and understanding in how health and the local environment interact from the research reported here, the quotation below represent the points made by the sample as a whole regarding the importance of clean air in relation to health:

GM4 (female): Here in Ust-Kamenogorsk, it's happening to all of us. We have little brothers and sisters, relatives and we worry about them. The last case of emissions was awful, everyone felt bad, half of all students felt bad at school.

GM1 (female): In summer, when it is hot, and there is no wind and everything is still, it's terrible. I mean people suffer with nasal pharynx, and indigestion. Many children are sick, and they were born sick already. (G11, CI, Oskemen city, east Kazakhstan)

\subsection{Physical Environment and Opportunities}

Free and easy access to parkland or other such leisure areas to include a variety of amenities were often identified as factors associated with increased levels of wellbeing. These findings are echoed within the literature [43] and confirm that young people's happiness and satisfaction with their environments is related to their overall wellbeing. Unfortunately, in this study many participants reported not only a lack of facilities in their local areas, but also expressed worries about their future educational and employment prospects. Young people living in small towns, remote rural areas as well as deprived urban areas often express these concerns. For example:

There is lack of places in our city where we can have rest or relax. There are several parks, but it is cold in the winter, and there is lack of special places for leisure. (Female, G9, CIII, Atyrau city, north-west Kazakhstan)

GM2 (female): There are some places to work, but they are not many interesting professions in our city.

Interviewer: So what are the typical professions here?

GM1 (male): Teachers and doctors and there is a railway, crushed stone plant and military plant in the city. (G10, CIII, Ereymentau town, northern-central Kazakhstan)

\subsection{Social Environment and Personal Safety (Home, Local Community, and School)}

In this study, young people identified feeling safe and secure as crucial to their 
wellbeing. The participants described a range of factors that could provide a sense of security and safety. These included having parents and friends that protect you and treat you well, as well as having a safe home, school, and local community. Unfortunately, many young people reported concerns pertaining to their personal safety and expressed feelings of helplessness in dealing and resolving these issues, further highlighting the negative impact on their wellbeing. For example:

GM5 (male): There is a prison not far from us. When a lot of people were freed from prison the crime rate increased rapidly in our city.

GM1 (female): Yes, we live in such awful district.

Interviewer: How does it influence your wellbeing?

GM5 (male): You hear news about what happens in the city, in families, different cases, and you start worrying and think how to protect your family and yourself. (G10, CIII, Oskemen city, east Kazakhstan)

In contrast, the general consensus among those interviewed in rural areas was the belief that they lived in harmony with most of their peers at school as well as with people in their local community. Young people from rural areas tended to describe strong bonds with their peers as well as adults in their community. A recurrent comment among these young people was that their peers were like their brothers and sisters, which was defined in the context of growing up together and knowing everyone well, further suggesting the development of strong bonds from early age. Another common comment was that their village was "a big family" and that they felt safe in their local community, with no major crimes and negative behaviors reported. For example:

In the village, everyone knows each other, no one fights and no-one takes drugs. Everyone knows each other in the village, has good relationships with each other and has a lot of friends. (Female, G11, CIV, Taraz city, southern Kazakhstan)

\subsection{Social Environment and More Generalized National and International Concerns (Peace, Harmony and Tolerance)}

Unfortunately, many young people expressed fears about war, terrorism, conflicts, the rise of extremist ideologies and financial crisis in Kazakhstan. These findings are in accord with previous studies that have highlighted young people's concerns and fears about their personal safety related to international conflicts, financial crises, and wars [44]. Young people in this study perceived themselves as helpless to do to anything about these issues, and called for society that is more tolerant. A tolerant society was defined in terms of unity, care, love, and respect for each other despite differences such as ethnicity, social status, disability, and religious beliefs. Some young people also expressed the need to change societal attitudes towards young people with disabilities and orphans living in Kazakhstan, while arguing for more inclusive societal approaches to reduce po- 
verty and inequality. These quotations represent the points made by the sample as a whole:

GM2 (male): I wish there was no strike in Kazakhstan, because of devaluation of tenge. One loaf of bread is 150 tenge, they say it can be 1000 tenge and people can rebel because of that, war can start because of that.

GM3 (male): Yes and here are some people who follow radical religions like Wahhabism. They are trying to distribute their ideas. They attract new members. Children, school students... (G11, CIV, Shymkent city, south Kazakhstan)

A lot of people suffer from wars and we need to help them. It is the same with disabled children or people, we can't ignore them. I think that our society should be more tolerant. (Female, G10, CI, Oskemen city, east Kazakhstan)

\subsection{Having Material Resources}

There is no dispute that the vast majority of young people interviewed were aware that money provides increased and inequitable access to cultural activities, social capital and educational opportunities. Having sufficient material resources was not only seen as having opportunities to purchase goods and services, but also portrayed as being able to provide a decent standard of living for families. Some participants discussed their own experiences of relative poverty and expressed concerns pertaining to the impact of poverty on their close relatives. Some accounts also highlighted the shame associated with poverty. These findings are echoed in literature exploring factors influencing young people's wellbeing [15] [43]. More importantly, in this study poverty was commonly linked to issue of corruption in Kazakhstan. These issues are commonly identified by young people in Central Asia as contributing towards a sense of insecurity in their society and highlighting feelings of oppression and economic exclusion. Money and corruption are often mentioned as pervading negative factors in educational establishments [44]. These quotations represent the points made by the sample as a whole:

Money plays an important role, even if the majority says that material values are not important. You need money for food, for relaxation, for simple things. (Male, G10, CII, Atyrau city, north-west Kazakhstan)

GM9 (male): What I can say about the Kazakhs economy is that the corruption is widespread. The corruption takes place in educational sphere too.

GM1 (female): Yes, some people even buy educational grants. (G10, CI, Oskemen city, east Kazakhstan)

In this section, the impact of external factors from one's surrounding on different aspects of wellbeing was discussed. The direct consequence on physical wellbeing from the inadequate and hostile physical environment as well as the environment's impact on psychological wellbeing through leisure, lifestyle, edu- 
cation, and employment opportunities was highlighted. Real and imminent concerns over personal and national safety and more abstract concerns over personal and national security were explored. This section also provided a unique insight into how material wealth interacts with both the environment and opportunities alongside perceived matters of social equity such as the effects of widespread corruption.

\section{The Quality of Relationships}

In this study, relationships featured strongly across all cohorts and were consistently considered by young people to be directly relevant to, and having impact on their wellbeing. The type, quality, and affection associated with relationships played an essential part in how young people reported their subjective wellbeing in a variety of contexts and situations. Far more important than a multiplicity of relationships (a specific number of friends) seemed to the perceived quality of relationships. There was a common agreement among participants that wellbeing can be enhanced through good quality of personal relationships based on values such as being reliable, available when needed, honest, fair as well as showing interest, respect, commitment and a willingness to listen to and taking the views of others seriously. These qualities were primarily listed in relation to family and friends, but it was apparent that young people also value them in their relations in schools and communities as highlighted in previous young people's wellbeing research [15] [36]. For example:

It is easy to make friends and you can find them everywhere. But it is the best friend who understands you, who puts you in a good mood, who makes your day, who tells you when you are wrong, and who shows you your mistakes. (Female, G10, CIII, Taraz city, southern Kazakhstan)

In this study, wellbeing was bound with the experience of being supported by others. Young people commonly linked social support with an increased ability to cope with stress and unfavorable situations. The participants also pointed out that support from relational network members was vital for achieving optimal levels of wellbeing. Based on young people's descriptions, support could be categorized into four main categories: informational (guidance or advice), instrumental (resources such as spending time with someone or providing them with materials or money), emotional (companionship, affection, and trust) and appraisal support (evaluative feedback and/or enhancement of self-worth). These findings are congruent with the social support literature that highlights young people's views of the significance of being supported, especially when dealing with unfavorable situations and/or transitioning into adulthood [45]. In addition, feelings of being supported, valued, and respected by others were identified by participants in this study as important psychological factors enabling more positive thinking and diminishing some of the negative aspects of daily life. For example: 
Failure affects your self-esteem. When you feel like a failure but your family gives you help and good advice you can change and achieve your goals. (Male, G10, CIII, Taraz city, southern Kazakhstan)

When you feel down your friends can cheer you up and help you, at least psychologically if not materially. (Male, G9, CII, Shymkent city, south Kazakhstan)

My home is a very important place for me. I know that there is always someone waiting for me and it makes me feel like I am wanted and needed.

(Female, G9, CI, Kokshetau city, northern Kazakhstan)

These findings are echoed within literature which documents that young people perceive stable and supportive relationships to be beneficial, as they provide encouragement, guidance and endorsement at key life events, as well as provide someone to turn to at points of crisis and change [45]. It has also been acknowledged that the experience of positive, safe and stable relationships can help young people to build secure attachments, develop self-confidence, self-esteem and self-reliance, thus contributing toward a strong sense of identity and belonging [46]. More importantly, if these foundations are in place, young people are afforded the best chance to secure positive long-lasting educational, health and overall wellbeing outcomes [47].

As can be gleaned from the references to familial ties above, family was clearly of paramount importance to young people's wellbeing. The majority of participants in this study described family as a place where one feels supported, respected, loved, valued, and included. Affirmative connections with relatives and a positive family climate were frequently associated with increased levels of emotional wellbeing. These findings confirm those of extant research which argues that parents can help their children to develop into emotionally healthy individuals by providing them with a supportive environment, affirmative feedback, and positive role modeling of healthy behavior and interactions [48].

Some participants also described the negative impact of family dysfunction such as divorce and parental alcohol abuse on the mental and emotional wellbeing of young people. Family conflict was frequently identified as important stressors in adolescents and a potential cause of health risk behaviors in youth. For example:

If the parents get divorced or consume alcohol their children might start drinking alcohol as well. Family has a big influence on the child (Female, G10, CII, Atyrau city, north-west Kazakhstan).

I don't like when my family is unhappy or tired it makes me stressed. (Female, G9, CI, Oskemen city, east Kazakhstan)

These findings are echoed within literature which highlights that families characterized by certain qualities are likely to have negative outcomes for mental health. These characteristics generally include overt family conflict, deficient nurturing, and family relationships that are cold, unsupportive, and neglectful. 
Families with these characteristics tend to leave their children vulnerable to a wide range of mental health problems such as anxiety, depression, and antisocial and health risk behaviors. More importantly, if vulnerable young people who struggle with mental health problems do not receive adequate and timely help, these issues are likely to exacerbate and become chronic and long-lasting [49].

In this section the perceived quality of relationships was identified as directly relevant to and having an impact on young people's wellbeing. The link between social support and an increased ability to cope with stress and unfavorable situations was highlighted. Affirmative connections with adults and peers were frequently associated with increased levels of emotional wellbeing.

\section{Self}

In this study, a positive sense of self was used through expressions of autonomy and individualism to define wellbeing by young people. Wellbeing seemed to be bound with experiences of being valued, experiences of oneself as a good person and aspiring to achieve, as echoed in study corroborated by Fattore et al. [15]. A sense of self-image tended to be grounded in reflections and feedback from others and feeling good about oneself was often linked with concrete achievements both inside and outside school life. The importance of making independent choices as well as being actively involved in leisure, volunteering and extracurricular school activities was highlighted by many participants and will be presented and discussed in further detail in the following sections.

\subsection{Ability to Make Independent Choices}

In this study, wellbeing was partly defined as the capacity to act freely, make choices, and exert influence in everyday situations, as reflected in wellbeing literature that highlights that young people often consider having choices and autonomy as concepts fundamental to their wellbeing [3] [15] [24]. The desire to make independent choices based on self-interest and personal wishes strongly featured among the older cohort. Some participants also pointed out constraints to their freedom by stating that they often felt obliged to fulfill their parents' wishes and reported that the decisions pertaining to their educational and career choices were guided and strongly influenced by their parents. For example:

I think the most negative thing is when you don't have a choice. In my case, my family tells me that I can do and what I should do so it is a bit of a problem for me. It brings my wellbeing down. (Female, G11, CI, Kostanay city, northern Kazakhstan)

The findings from this study support those of extant research which suggests that the transition to adulthood implies a regulation of distance within the family, described as a modification of the balance between connectedness and autonomy. The underlining assumption shared by researchers is that the transition to adulthood does not imply a disruption of family bonds but a transformation 
in the direction of greater interdependence [50]. In addition, a growing sense of autonomy is often identified as an important factor enabling young people to develop towards adulthood, where opportunities to make decisions with support are considered to positively contribute towards this process [51]. However, it is important to acknowledge that cultures, particularly in central Asia, tend to value interdependence such as collective achievement, sharing, and collaboration. These values often are expressed in parental expectations that children obey authority, share their possessions, and place the family's and community's needs before their own [52].

\subsection{Doing Good Deeds (Altruism)}

In this study, many participants reported that enhancing self-worth and personal effectiveness by helping others through volunteering (usually organized via school as a part of extra-curricular school activities) was important to their wellbeing. Carrying out good deeds was commonly linked to feelings of happiness, as it felt good to help others in need, especially those who were perceived as less fortunate. Some participants noted that being a volunteer was not only beneficial for the person in need but also that through helping others they often acquired new skills that could potentially benefit them in the future. More importantly, helping others appeared to provide young people with a real sense of perspective, often highlighted in their accounts describing "how lucky they were", and suggesting a more positive outlook on the things that were causing them stress. When young people living in rural areas were asked about their leisure activities they pointed out having limited time for hobbies due to chores such as housework, caring for younger siblings and farm animals. However, they did not seem to perceive doing the chores as constraints on their freedom as their views highlighted a certain level of personal satisfaction related to feelings of being useful and helpful, further highlighting the positive impact of altruism on young people's wellbeing. These quotations represent the points made by the sample as a whole:

I love reading aloud for my little sisters. I also like doing sport, especially wrestling, and planting willows in our garden. I love riding horses and taking care of our animals. (Male, G9, CII, Shymkent city, south Kazakhstan) I feel good about myself when I can help other people. It's good thing when we do something for others, because we can help and we can learn new things. (Female, G9, CI, Kokshetau city, northern Kazakhstan)

These findings are further supported by research evidence suggests that altruistic attitudes, volunteering, and informal helping behaviors can make unique contributions to the maintenance of life satisfaction and positive affect [53] [54]. Involvement in pro-social activities can reduce stress as well as improve mood, self-esteem, and happiness. Helping can also improve social support, encourage young people to lead a more physically active lifestyle, and distract from person- 
al problems, hence allowing engagement in a meaningful activity and improvement in self-esteem and competence. Being part of a social network might increase feelings of belonging and can help reduce loneliness and isolation. An act of kindness can improve confidence, control, happiness and optimism. It can also indirectly encourage others to repeat the good deed and thus contribute to a more positive community [54].

\subsection{Being Active}

There was no dispute that active participation in leisure activities was important for young people's wellbeing. When participants were asked to name factors they believed could enhance their own wellbeing the majority of them included in their descriptions at least one leisure activity. The range of leisure activities described was very diverse. The most common leisure activities reported by young people were listening to music, sport, creative, visual and performing arts, caring for pets, reading, walking in the nature, internet, social media, and watching TV. Some of the participants also pointed out that there were many leisure activities they would like to be involved in, but required payment that many parents could not afford, which according to their own words made them upset. It should also be noted that many participants attending selective schools noted that they not only lacked free time due to the high workload, but they also felt that some of the school staff members showed negative attitudes towards activities that they perceived as not academically beneficial.

Based on the participants' accounts leisure activities were commonly associated with sensory experiences such as physical exhilaration in sports, dance, and/or visual responses to creativity. Some young participants also described how activities in which they achieved success evoked a sense of recognition and appreciation, commonly associated with approbation from their family and friends, further suggesting a contribution to their sense of competence. Being good at something also appeared to be an important aspect of young people's identities and of self-esteem. Their accounts also highlighted that being actively engaged in leisure activities that were perceived by the participants as meaningful and beneficial played an integral role in building, enhancing, and maintaining social relationships with their peers and family. In addition, some participants portrayed leisure activities such as music and/or reading as a useful tool in obtaining desired levels of cognitive stimulation and inspiration. For example:

I like reading books including extracurricular books. They are interesting, they increase your knowledge and self-confidence. (Female, G9, CII, Atyrau city, north-west Kazakhstan)

Expressing feelings through active engagement in performing and visual arts seemed to play an important role in the lives of young people, featuring strongly for the older cohort especially. Self-expression through art seemed to generate personal exploration as well as feelings of being heard, and also linked to expe- 
riencing freedom. For example:

Art is like a means of self-expression for me, because a lot of people can't understand my point of view. Through illustrations, through art or some simple kind of dance, I try to do all those things. I can tell something to people and they will understand me, even if we speak different languages.

(Female, G11, CII, Shymkent city, south Kazakhstan)

Emotional regulation through active engagement in desired leisure activities also seemed to play an important role in young people's emotional lives, potentially providing the means through which wellbeing could be enhanced and restored. The role of music was often described through its role in modifying negative cognitions, assisting rational thinking about life issues, and inspiring creativity. Some of the participants' accounts also suggested that the use of music could help to modify young people's emotions and levels of arousal when these were undesirable or discordant with current goals and psychological needs pertinent to their wellbeing. These findings are in congruence with previous studies that highlight that physical exercise and music are commonly used as a mood regulation strategy by young people, further arguing that these activities can have great potential to assist in achieving optimal functioning and wellbeing [55]. In addition, listening to one's music of choice was often portrayed by young people in this study as a distraction from negative or undesirable feelings and thoughts. For example:

Music helps me when I am a bad mood and I can't share it with my friends or parents. It puts me in a good mood. (Female, G10, CIII, Taraz city, southern Kazakhstan)

Being actively involved in challenging and intrinsically motivating leisure activities is considered to be a vital part of the development of youth. According to a study corroborated by Larson and Rusk [56], active participation in meaningful leisure activities can provide context in which young people are likely to develop skills and competencies, form friendships and relationships, achieve mental and physical health, express creativity, develop a self-identity, and determine meaning and purpose in life.

\subsection{Extracurricular Activities via School}

Many young people pointed out that their schools provided free accesses to their facilities via extra-curricular activities that were organized by teachers and social educators employed by the school. Incorporating extra activities and clubs in school was commonly described as beneficial for young people's overall wellbeing. The benefits of extracurricular activities to young people's wellbeing are commonly highlighted in youth development studies [57] [58]. These studies point out that well-structured extracurricular activities can enable young people to develop and practice valuable social, emotional, and physical skills, develop a better sense of agency as a member of one's community, as well as develop social 
support networks of peers and adults that might become a helpful resource in the present and future. In turn, assets acquired through participation in extra-curricular activities might facilitate increased school engagement and provide a protective context in terms of risky behaviors as well as a promotive context in terms of academic performance.

In this section, a positive sense of self was bound with experiences of being valued, seeing oneself as a good person, and aspiring to achieve. A sense of self-image was grounded in reflections and feedback from others and feeling good about oneself via concrete achievements both inside and outside school life. The importance of making independent choices as well as being actively involved in leisure, volunteering and extracurricular school activities for achieving optimal levels of wellbeing was highlighted.

\section{Conclusions}

The data represents the views of 309 young people on their conceptualizations of wellbeing, which to our knowledge could be considered the first wellbeing research of this nature conducted in Kazakhstan. The study results also clearly demonstrate young people's full capacity to not only define, but also deconstruct the complex topic of wellbeing as highlighted in previous research [10] [15] [36]. Such evidence contributes to the argument that young people can not only provide unique insights into their lifeworlds but also make meaningful contributions to understandings of wellbeing, thus extending the current knowledge base.

Findings reported in this article might be seen as presenting a problem-focused perspective. However, strength-based approaches also require the identification of problems in order to activate solutions that facilitate the self-esteem of individuals and power communities [59]. A key part of a strength-based approach is also the recognition that some people might think of a problem as an integral part of their character. Hence separating this problem from the individual by externalizing it might allow them to deal with it in a more constructive manner [60]. Therefore further research might explore the "deficit areas" identified by young people in this study by the use of narrative inquiry. It has been argued that story telling methodology, which is based on the assumption that inside any problem narrative is a story of strengths and resilience, has the potential to develop and maintain an individual's hope, a concept that is critical to recovery and thus increased levels of wellbeing [61].

In this study, listening to young people's voices also uncovered a large number of components young people perceived as affecting their wellbeing in many different ways. This new knowledge can potentially help facilitate the development of more comprehensive measurements and indicators that are relevant to young people in Kazakhstan, thus allowing clear targets to be created for effectively supporting wellbeing. Importantly, it also has the potential to inform the development of tools that can provide on-going opportunities for young people to 
self-report on their sense of wellbeing both inside and outside the school context. The findings also indicate variations in the value attributed to different contextual factors contributing to wellbeing within our sample of participants. Therefore, in order to provide a more complex and comprehensive picture of young people's wellbeing, further analysis is required to identify the extent to which the differences in values are related to socio-economic attributes such as socioeconomic background, ethnicity, and gender. The way forward in improving the wellbeing of young people is for practitioners from other fields and policy-makers to work collaboratively to address the concerns raised by the participants.

This paper also provides guidance and we trust further motivation for researchers and practitioners to rise to the challenge of providing young people the opportunity to contribute to the discussions about their wellbeing. Even though this paper focuses solely on perspectives of young people and their conceptualization of wellbeing, it is not proposed that young people's views should be sought at the exclusion or detriment of adult's voices and opinions. Instead, it is crucial to include perspectives of all stakeholders to ensure that policy, services and provisions are appropriately meeting the needs of those involved.

\section{Acknowledgements}

This research was funded by the "Newton-Al Farabi Partnership Programme" (Reference number 172734464). The Newton-Al Farabi Partnership Programme is coordinated by JSC Science Fund, for Kazakhstan, and the British Council, for the United Kingdom. We would like to acknowledge the full research team for their contribution to this paper: Carole Faucher, Daniel Hernández-Torrano, Anna Sioni CohenMiller, Kairat Kurakbayev, Ainur Almukhambetova, Madina Tynybayeva, and Assel Batyrova. We would also like to thank the important work undertaken by researchers from Nazarbayev University Graduate School of Education.

\section{References}

[1] Ben-Arieh, A. (2006) Measuring and Monitoring the Well-Being of Young Children around the World. Background Paper Prepared for the Education for All Global Monitoring Report 2007. Strong Foundations: Early Childhood Care and Education.

[2] Bradshaw, J., Keung, A., Rees, G. and Goswami, H. (2011) Children's Subjective Well-Being: International Comparative Perspectives. Children and Youth Services Review, 33, 548-556.

[3] Bradshaw, J. and Richardson, D. (2009) An Index of Child Well-Being in Europe. Child Indicators Research, 2, 319-351. https://doi.org/10.1007/s12187-009-9037-7

[4] Diener, E. and Seligman, M.E.P. (2004) Beyond Money: Toward an Economy of Well-Being. Psychological Science in the Public Interest, 5, 11-31. https://doi.org/10.1111/j.0963-7214.2004.00501001.x

[5] Huppert, F., Marks, N., Clark, A., Siegrist, J., Stutzer, A., Vittersø, J. and Wahrendorf, M. (2009) Measuring Well-Being across Europe: Description of the ESS 
Well-Being Module and Preliminary Findings. Social Indicators Research, 91, 301-315. https://doi.org/10.1007/s11205-008-9346-0

[6] New Economics Foundation (2009) National Accounts of Well-Being. http://www.nationalaccountsofwellbeing.org/learn/measuring/

[7] United Nations (2009) The Millennium Development Goals Report. United Nations, New York.

[8] Soutter, A.K., O’Steen, B. and Gilmore, A. (2014) The Student Well-Being Model: A Conceptual Framework for the Development of Student Well-Being Indicators. International Journal of Adolescence and Youth, 19, 496-520. https://doi.org/10.1080/02673843.2012.754362

[9] Coombes, L., Appleton, J.V., Allen, D. and Yerrell, P. (2013) Emotional Health and Well-Being in Schools: Involving Young People. Children and Society, 27, 220-232. https://doi.org/10.1111/j.1099-0860.2011.00401.x

[10] Crivello, G., Camfield, L. and Woodhead, M. (2009) How Can Children Tell Us about Their Wellbeing? Exploring the Potential of Participatory Research Approaches within Young Lives. Social Indicators Research, 90, 51-72.

https://doi.org/10.1007/s11205-008-9312-x

[11] Mashford-Scott, A., Church, A. and Tayler, C. (2012) Seeking Children's Perspectives on Their Wellbeing in Early Childhood Settings. International Journal of Early Childhood, 44, 231-247. https://doi.org/10.1007/s13158-012-0069-7

[12] Pollard, E.L. and Lee, P.D. (2003) Child Well-Being: A Systematic Review of the Literature. Social Indicators Research, 61, 59-78.

https://doi.org/10.1023/A:1021284215801

[13] Camfield, L., Streuli, N. and Woodhead, M. (2009) What's The Use of 'Well-Being' in Contexts of Child Poverty? Approaches to Research, Monitoring and Children's Participation. International Journal of Children's Rights, 17, 65-109. https://doi.org/10.1163/157181808X357330

[14] Watson, D., Emery, C. and Bayliss, P. (2012) Children's Social and Emotional Wellbeing. The Policy Press, Bristol. https://doi.org/10.2307/j.ctt1t89923

[15] Fattore, T., Mason, J. and Watson, E. (2007) Children's Conceptualisation (s) of Their Well-Being. Social Indicators Research, 80, 5-29.

https://doi.org/10.1007/s11205-006-9019-9

[16] McAllister, F. (2005) Wellbeing: Concepts and Challenges. Discussion Paper Prepared for the Sustainable Development Research Network.

http://www.sdresearch.org.uk/wellbeing/documents/SDRNwellbeingpaper-Final_00 0.pdf

[17] Nastasi, B.K. and Borja, A.P., Eds. (2016) International Handbook of Psychological Well-Being in Children and Adolescents: Bridging the Gaps between Theory, Research, and Practice. Springer Science and Business Media, New York. https://doi.org/10.1007/978-1-4939-2833-0

[18] Roelen, K. and Gassmann, F. (2014) Beyond Averages: Child Well-Being in Kazakhstan. Journal of Children and Poverty, 20, 91-110. https://doi.org/10.1080/10796126.2014.979139

[19] CohenMiller, A.S., Faucher, C., Hernández-Torrano, D. and Hajdukova, E.B. (2017) Practical Steps for Using Interdisciplinary Educational Research to Enhance Cultural Awareness. International Journal of Research and Method in Education, 40, 288-298. https://doi.org/10.1080/1743727X.2017.1310834

[20] Knox, C. (2008) Kazakhstan: Modernizing Government in the Context of Political 
Inertia. International Review of Administrative Sciences, 74, 477-496. https://doi.org/10.1177/0020852308095314

[21] Castells, M. (2011) The Power of Identity: The Information Age: Economy, Society, and Culture. Blackwell Publishing, Oxford.

[22] Mukhtar, A. (2015) Changing Values in the Context of Generational Approach in the Kazakhstan. Asian Social Science, 11, 181-191. https://doi.org/10.5539/ass.v11n14p181

[23] Inoguchi, T. (2015) Multiple Modes of Wellbeing in Asia. In: Global Handbook of Quality of Life, Springer, Netherlands, 597-607. https://doi.org/10.1007/978-94-017-9178-6_27

[24] Anderson, D.L. and Graham, A.P. (2016) Improving Student Wellbeing: Having a Say at School. School Effectiveness and School Improvement, 27, 348-366. https://doi.org/10.1080/09243453.2015.1084336

[25] Soutter, A.K., O’Steen, B. and Gilmore, A. (2014) The Student Well-Being Model: A Conceptual Framework for the Development of Student Well-Being Indicators. International Journal of Adolescence and Youth, 19, 496-520. https://doi.org/10.1080/02673843.2012.754362

[26] Sixsmith, J., Gabhainn, N.S., Fleming, C. and O'Higgins, S. (2007) Childrens', Parents' and Teachers' Perceptions of Child Wellbeing. Health Education, 107, 511-523. https://doi.org/10.1108/09654280710827911

[27] Ruck, M.D., Keating, D.P., Saewyc, E.M., Earls, F. and Ben-Arieh, A. (2014) The United Nations Convention on the Rights of the Child: Its Relevance for Adolescents. Journal of Research on Adolescence, 26, 16-29. https://doi.org/10.1111/jora.12172

[28] Taylor, R.L., Olds, T., Boshoff, K. and Lane, A.E. (2010) Children's Conceptualization of the Term 'Satisfaction': Relevance for Measuring Health Outcomes. Child: Care, Health and Development, 36, 663-669. https://doi.org/10.1111/j.1365-2214.2010.01105.x

[29] Patton, M.Q. (2005) Qualitative Research. Qualitative Research. Sage, Thousand Oaks, CA.

[30] McLellan, R. and Steward, S. (2015) Measuring Children and Young People's Wellbeing in the School Context. Cambridge Journal of Education, 45, 307-332. https://doi.org/10.1080/0305764X.2014.889659

[31] Temple, B. and Young, A. (2004) Qualitative Research and Translation Dilemmas. Qualitative Research, 4, 161-178. https://doi.org/10.1177/1468794104044430

[32] Punch, S. (2002) Research with Children: The Same or Different from Research with Adults? Childhood, 9, 321-341. https://doi.org/10.1177/0907568202009003005

[33] Sapkota, P. and Sharma, J. (1996) Participatory Interactions with Street Children in Nepal. PLA Notes, 25, 61-64.

[34] Edwards-Jones, A. (2014) Qualitative Data Analysis with NVIVO. Journal of Education for Teaching, 40, 193-195. https://doi.org/10.1080/02607476.2013.866724

[35] Braun, V. and Clarke, V. (2013) Successful Qualitative Research: A Practical Guide for Beginners. Sage, London.

[36] Simmons, C., Graham, A. and Thomas, N. (2014) Imagining an Ideal School for Wellbeing: Locating Student Voice. Journal of Educational Change, 16, 129-144. https://doi.org/10.1007/s10833-014-9239-8

[37] Pople, L., Rees, G., Main, G. and Bradshaw, J. (2015) The Good Childhood Report 
2015. The Children' Society, London.

http://www.childrenssociety.org.uk/sites/default/files/TheGoodChildhoodReport20 15.pdf

[38] Ben-Arieh, A. and Frønes, I. (2011) Taxonomy for Child Well-Being Indicators: A Framework for the Analysis of the Well-Being of Children. Childhood, 18, 460-476. https://doi.org/10.1177/0907568211398159

[39] United Nations Children's Fund (2007) Child Poverty in Perspective: An Overview of Child Wellbeing in Rich Countries. https://www.unicef.org/infobycountry/files/ChildPovertyReport.pdf

[40] Mitchell, R. and Popham, F. (2008) Effect of Exposure to Natural Environment on Health Inequalities: An Observational Population Study. The Lancet, 372, 1655-1660.

[41] Kenessariyev, U., Golub, A., Brody, M., Dosmukhametov, A., Amrin, M., Erzhanova, A. and Kenessary, D. (2013) Human Health Cost of Air Pollution in Kazakhstan. Journal of Environmental Protection, 4, 1-8. https://doi.org/10.4236/jep.2013.48101

[42] OECD (2016) Multi-Dimensional Review of Kazakhstan: Initial Assessment. OECD Publishing, Paris.

[43] Rees, G., Goswami, H. and Pople, L. (2013) The Good Childhood Report 2013. http://citeseerx.ist.psu.edu/viewdoc/download?doi=10.1.1.394.7901\&rep=rep1\&type $=\mathrm{pdf}$

[44] Paasiaro, M., Quinn-Judge, K. and Arynov, M. (2012) Nobody Has Ever Asked about Young People's Opinions: Young People’s Perspectives on Identity, Exclusion and the Prospects for a Peaceful Future in Central Asia. Saferworld, London.

[45] Singer, E.R., Berzin, S.C. and Hokanson, K. (2013) Voices of Former Foster Youth: Supportive Relationships in the Transition to Adulthood. Children and Youth Services Review, 35, 2110-2117. https://doi.org/10.1016/j.childyouth.2013.10.019

[46] Currie, C., Zanotti, C., Morgan, A., et al., Eds. (2012) Social Determinants of Health and Well-Being among Young People. Health Behaviour in School-Aged Children (HBSC) Study: International Report from the 2009/2010 Survey. World Health Organization, Copenhagen.

[47] Siebelt, L., Morrison, E. and Cruickshank, C.A. (2008) Caring about Success: Young People's Stories. Edinburgh, Scotland.

[48] Morris, A.S., Silk, J.S., Steinberg, L., Myers, S.S. and Robinson, L.R. (2007) The Role of the Family Context in the Development of Emotion Regulation. Social Development, 16, 361-388. https://doi.org/10.1111/j.1467-9507.2007.00389.x

[49] Jose, P.E., Ryan, N. and Pryor, J. (2012) Does Social Connectedness Promote a Greater Sense of Well-Being in Adolescence Over Time? Journal of Research on Adolescence, 22, 235-251. https://doi.org/10.1111/j.1532-7795.2012.00783.x

[50] Masten, A.S., Burt, K.B., Roisman, G.I., Obradovic, J., Long, J.D. and Tellegen, A. (2004) Resources and Resilience in the Transition to Adulthood: Continuity and Change. Development and Psychopathology, 16, 1071-1094. https://doi.org/10.1017/S0954579404040143

[51] Hare, A.L., Szwedo, D.E., Schad, M.M. and Allen, J.P. (2015) Undermining Adolescent Autonomy with Parents and Peer: The Enduring Implications of Psychologically Controlling Parenting. Journal of Research on Adolescence, 25, 739-752. https://doi.org/10.1111/jora.12167

[52] Greenfield, P.M. (2009) Linking Social Change and D elopmental Change: Shifting 
Pathways of Human Development. Developmental Psychology, 45, 401-418. https://doi.org/10.1037/a0014726

[53] Kahana, E., Bhatta, T., Lovegreen, L.D., Kahana, B. and Midlarsky, E. (2013) Altruism, Helping, and Volunteering: Pathways to Well-Being in Late Life. Journal of Aging and Health, 25, 159-187. https://doi.org/10.1177/0898264312469665

[54] Benson, P.L., Clary, E.G. and Scales, P.C. (2007) Altruism and Health: Is There a Link during Adolescence? In: Post, S.G., Ed., Altruism and Health: Perspectives from Empirical Research, Oxford University Press, New York, 97-115. https://doi.org/10.1093/acprof:oso/9780195182910.003.0009

[55] MacDonald, R.A.R., Kreutz, G. and Mitchell, L. (2012) Music, Health and Wellbeing. Oxford University Press, Oxford. https://doi.org/10.1093/acprof:oso/9780199586974.001.0001

[56] Larson, R.W. and Rusk, N. (2011) Intrinsic Motivation and Positive Development. In: Lerner, R.M., Lerner, J.V. and Benson, J.B., Eds., Advances in Child Development and Behavior: Positive Youth Development, England: Elsevier, Oxford, 89-130. https://doi.org/10.1016/B978-0-12-386492-5.00005-1

[57] Eccles, J.S., Barber, B.L., Stone, M. and Hunt, J. (2003) Extracurricular Activities and Adolescent Development. Journal of Social Issues, 59, 865-889. https://doi.org/10.1046/j.0022-4537.2003.00095.x

[58] Park, N. (2004) The Role of Subjective Well-Being in Positive Youth Development. The Annals of the American Academy of Political and Social Science, 591, 25-39. https://doi.org/10.1177/0002716203260078

[59] Lopez, S.J. and Louis, M.C. (2009) The Principles of Strengths-Based Education. Journal of College and Character, 10, 1-8. https://doi.org/10.2202/1940-1639.1041

[60] Epston, D. and White, M. (1992) Consulting Your Consultants: The Documentation of Alternative Knowledges. In: Epston, D. and White, M., Eds., Experience, Contradiction, Narrative and Imagination: Selected Papers of David Epston and Michael White, Australia: Dulwich Centre, Adelaide, 11-26.

[61] Smock, S.A., Trepper, T.S., Wetchler, J.L., McCollum, E.E., Ray, R. and Pierce, K. (2008) Solution-Focused Group Therapy for Level 1 Substance Abusers. Journal of Marital and Family Therapy, 34, 107-120. https://doi.org/10.1111/j.1752-0606.2008.00056.x 


\section{Appendix 1}

Drawing activity description (used as a tool to initiate discussion about wellbeing):

Instructions:

- Draw 5 items/concepts that represent things that you believe have a positive influence on your wellbeing, making you feel positive and happy.

- Draw 5 items/concepts that represent things that you believe have negative influence on your wellbeing, making you feel unhappy.

- Please name each of the items/things you draw and explain why you have chosen to draw it.

Examples of the participants' pictures:
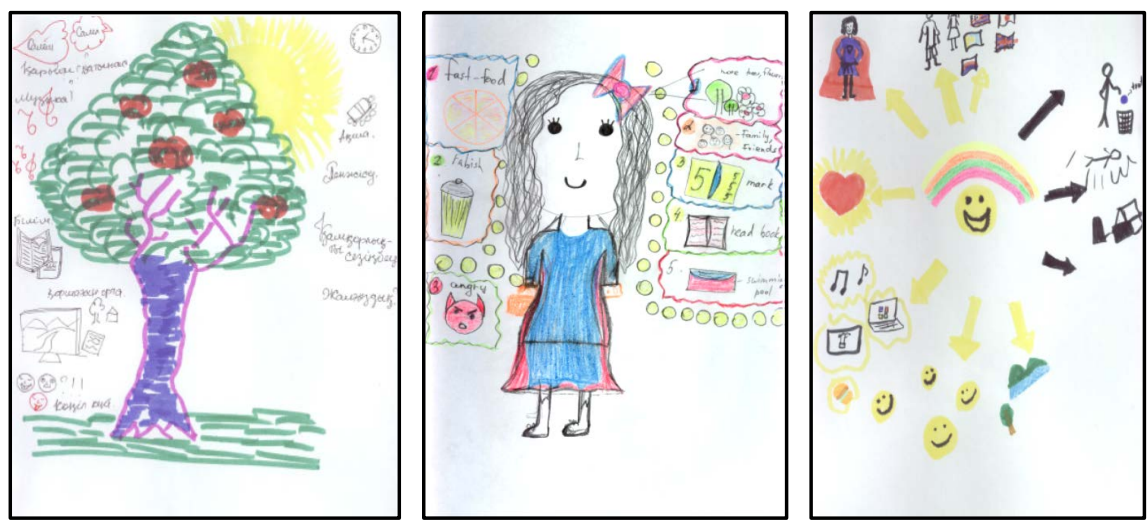

\section{Interview protocol: Question examples}

What does wellbeing mean to you?

What do you think all children and young people need for a good life?

What do you think all children and young people need for a healthy life?

What do you have in your life that you wish others could have as well?

What would you change about your life if you could?

What can stop young people and children from having a happy and healthy life?

What do you think makes children and young people feel happy?

Who would you talk to if you were having a hard time or if you were worried about your friends? 